M 1 Raabe Einfübrung in die Bücherkunde

M 3 Meisen Altdeutsche Grammatik II: Formenlebre

M 4 Grimm Bertolt Brecht

M 5 Moser Annalen der deutschen Sprache

M 6 Schlawe. Literarische Zeitschriften 1885-1910

M 7 Weber/Hoffmann Nibelungenlied

M 8 Meyer Eduard Mörike

M 9 Rosenfeld Legende

M 10 Singer Der galante Roman

M 12 Nagel Meistersang

M 13 Bangen Die schriftliche Form germanist. Arbeiten

M 14 Eis Mittelalterliche Fachliteratur

M 15 Weber/Hoffmann Gottfried von Straßburg

M 16 Lüthi Märchen

M 17 Wapnewski Hartmann von Aue

M 18 Meetz Friedrich Hebbel

M 19 Schröder Spielmannsepik

M 20 Ryan Friedrich Hölderlin

M 22 Danzel Zur Literatur und Philosophie der Goethezeit

M 24 Schlawe Literarische Zeitschriften 1910-1933

M 25 Anger Literarisches Rokoko

M 26 Wodtke Gottfried Benn

M 27 von Wiese Novelle

M 28 Frenzel Stoff-, Motiv- und Symbolforschung

M 29 Rotermund Christian Hofmann von Hofmannswaldau

M 30 Galley Heinrich Heine

M 31 Müller Franz Grillparzer

M 32 Wisniewski Kudrun

M 33 Soeteman Deutsche geistliche Dichtung des 11. u. 12. Jh.s

M 34 Taylor Melodien des Mittelalters I: Darstellung

M 35 Taylor Melodien des Mittelalters II: Materialien

M 36 Bumke Wolfram von Eschenbach

M 37 Engel Handlung, Gespräch u. Erzäblung. Faksimiledruck

M 38 Brogsitter Artusepik

M 39 Blanckenburg Versuch über den Roman. Faksimiledruck

M 40 Halbach Walther von der Vogelweide

M 41 Hermand Literaturwissenschaft und Kunstwissenschaft

M 43 Glinz Deutsche Syntax 


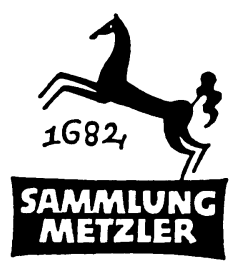

REALIEN ZUR LITERATUR

ABT. D:

LITERATURGESCHICHTE 


\section{Die Literatur des}

\section{deutschen Frühhumanismus}


CIP-Kurztitelaufnahme der Deutschen Bibliothek

Bernstein, Eckhard

Die Literatur des deutschen Frühhumanismus. -

1. Aufl. - Stuttgart: Metzler, 1978.

(Sammlung Metzler; M 168: Abt. D, Literaturgeschichte)

ISBN 978-3-476-10168-6

ISBN 978-3-476-10168-6

ISBN 978-3-476-03874-6 (eBook)

DOI 10.1007/978-3-476-03874-6

M 168

(C) Springer-Verlag GmbH Deutschland 1978

Ursprünglich erschienen bei J. B. Metzlersche Verlagsbuchhandlung und Carl Ernst Poeschel Verlag GmbH in Stuttgart 1978 


\section{VORBEMERKUNG}

Ziel der folgenden Darstellung ist es, eine auf Fakten beruhende Einführung in die Literatur des deutschen Frühhumanismus zu geben. Obwohl diese Literatur überwiegend in der Volkssprache verfaßt wurde, wäre eine Beschränkung auf deutschsprachige literarische Produkte weder sinnvoll noch gerechtfertigt. Sie würde in unzulässiger Weise das Bild der Epoche verfälschen.

Arbeiten der Sekundärliteratur, soweit sie für den Frühhumanismus von allgemeiner Bedeutung sind und sich nicht ausschließlich mit einem Einzelproblem oder Einzelautor beschäftigen, werden zu Beginn in alphabetischer Reihenfolge aufgeführt. Hinweise im Text beziehen sich stets auf dieses Verzeichnis.

Dem College of the Holy Cross sei für die Gewährung eines vorlesungsfreien Forschungsjahres, während dessen diese Arbeit entstand, herzlichst gedankt.

Worcester, Massachusetts 1977

E. B. 
Literaturverzeichnis . . . . . . . . . . . VIII

I. Einleitung . . . . . . . . . . . . . . 1

II. Voraussetzungen . . . . . . . . . . . . . 5

1. Der italienische Humanismus . . . . . . . . . 5

2. Die Begriffe "Studia humanitatis«, "Humanist» und "Humanismus" . . . . . . . . . . . . 7

3. Enea Silvio Piccolomini als Vermittler . . . . . 9

III. Die Entwicklung des Humanismus in Deutschland . 14

Wien (15) - Heidelberg (18) - Basel (20) - Augsburg (22) - Nürnberg (26) - Ingolstadt (29) - Erfurt (31) - Leipzig (32)

IV. Zwei frühe humanistische Propagandoren . . . . . 34

1. Peter Luder . . . . . . . . . . . . . . . . 34

2. Samuel Karoch von Lichtenberg . . . . . . . 38

V. Frühhumanistische Übersetzer . . . . . . . . 41

1. Niclas von Wyle . . . . . . . . . . . 43

a) Biographie . . . . . . . . . . . 43

b) Werke ............... . 46

c) Ubersetzungsprinzipien und Wirkung . . . . 60

2. Albrecht von Eyb . . . . . . . . . . . . 62

a) Biographie ............ . . 63

b) Werke ............... 65

3. Heinrich Steinhöwel . . . . . . . . 75

a) Biographie . . . . . . . . . . . 76

b) Werke . . . . . . . . . . . . 77

4. Andere Übersetzer . . . . . . . . . . . . . 91

a) Heinrich Schlüsselfelder . . . . . . . . . 91

b) Konrad Humery . . . . . . . . . . . . . 93

c) Ludwig Hohenwang . . . . . . . . . . . 94

d) Hans Neithart . . . . . . . . . . . 96

VI. Probleme und Aufgaben der Forschung . . . . . . 99

Register . . . . . . . . . . . 103 
ADB

AfdA

AKG

AfKdV

Archiv

BLV

DA

DVjs

GRM

$\mathrm{HJ}$

$\mathrm{HZ}$

JbiG

JEGP

MLN

MLR

PBB (Halle)

PBB (Tüb.)

RL

RQ

VL

WZUG

ZfdA

$\mathrm{ZfdPh}$

ZGO

ZvglLg

ZWLg
Allgemeine Deutsche Biographie

Anzeiger für deutsches Altertum

Archiv für Kulturgeschichte

Anzeiger für Kunde der deutschen Vorzeit

Archiv für das Studium der neueren Sprachen und Literaturen

Bibliothek des Literarischen Vereins in Stuttgart

Dissertation Abstracts

Deutsche Vierteljahrsschrift für Literaturwissenschaft und Geistesgeschichte

Germanisch-romanische Monatsschrift

Historisches Jahrbuch

Historische Zeitung

Jahrbuch für internationale Germanistik

Journal of English and Germanic Philology

Modern Language Notes

Modern Language Review

Beiträge zur Geschichte der deutschen Sprache und Literatur (Halle)

Beiträge zur Geschichte der deutschen Sprache und Literatur (Tübingen)

Reallexikon der deutschen Literaturgeschichte

Renaissance Quarterly

Die deutsche Literatur des Mittelalters: Verfasser-

lexikon

Wissenschaftliche Zeitschrift der Ernst-Moritz-

Arndt-Universität Greifswald

Zeitschrift für deutsches Altertum

Zeitschrift für deutsche Philologie

Zeitschrift für die Geschichte des Oberrheins

Zeitschrift für vergleichende Literaturgeschichte

Zeitschrift für Württembergische Landesgeschichte 


\section{LITERATURVERZEICHNIS}

\section{Bibliographien und Forschungsberichte}

Bibliographie internationale de l'Humanisme et de la Renaissance, Genf (erscheint jährlich seit 1966).

Engel, J. E.: Zeitalter der Renaissance, des Humanismus und der Reformation, in: Handbuch der dt. Lit.-Geschichte, Abt. Bibliographien, Bd. 4, 1969.

Fischer, H.: Neue Forschungen zur deutschen Dichtung des Spätmittelalters (1230-1500), in: DVjs 31, 1957, S. 303-345.

ders.: Probleme und Aufgaben der Literaturforschung zum deutschen Spätmittelalter, in: GRM 40, 1959, S. 217-227.

Herding, O.: Uber einige Richtungen in der Erforschung des deutschen Humanismus seit etwa 1945, in: Humanismusforschung seit 1945, 1975.

Janota, J.: Neue Forschungen zur deutschen Dichtung des Spätmittelalters (1230-1500), in: DVjs 45, 1971, S. 1-242.

Newald, R.: Deutsche Literatur im Zeitalter des Humanismus. Ein Literaturbericht, in: DVjs 27, 1953, S. 309-326.

Nolte, F. O.: German Literature and the Classics: a Bibliographical Guide, in: Harvard Studies in Philology 18, 1935, S. 125-163.

Spitz, L. W.: German Humanism, in: RQ 21, 1968, S. 125-131.

Taylor, A.: Problems in German Literary History of the Fifteenth and Sixteenth Centuries, New York 1939.

Thompson, L. S.: German Translations of the Classics between 1450-1500, in: JEGP 42, 1943, S. 343-363.

Worstbrock, F. J.: Deutsche Antikerezeption 1450-1550. I, Verzeichnis der deutschen Ubersetzungen antiker Autoren mit einer Bibliographie der Ubersetzer, 1976.

Wuttke, D.: Deutsche Germanistik und Renaissance-Forschung. Ein Vortrag zur Forschungslage, 1968.

\section{Sekundärliteratur}

Baechtold, J.: Geschichte der deutschen Literatur in der Schweiz, Frauenfeld 1892.

Baron, F. E.: The Beginnings of German Humanism. The Life and Work of the Wandering Humanist Peter Luder, Ann Arbor, Michigan 1967.

Beyschlag, S.: Städte, Höfe, Gelehrte, 1430-1490, in: Annalen der deutschen Literatur, hg. v. H. O. Burger, 1971.

Bidlingmaier, E. E.: Die sprachgeschichtliche Stellung der schwäbischen Frühhumanisten, in: Mitt. d. Vereins f. Kunst u. Altertum in Ulm u. Oberschwaben 28, 1932, S. 22-31.

Bobertag, F.: Geschichte des Romans und der ihm verwandten Dichtungsgattungen, I, 1881.

Boeck, J. G.: Geschichte der deutschen Literatur von 1480-1600, 
1961 (Gesch. der dt. Lit., hg. v. Kollektiv der Literaturgeschichte, Bd. 4).

Buck, A.: Die studia humanitatis und ihre Methode, in: Bibliothèque d'Humanisme et Renaissance 21, 1959, S. 273-290.

ders.: Der italienische Humanismus, in: Humanismusforschung seit 1945, 1975.

Bübler, C. F.: The Fifteenth-Century Book. The Scribes, the Printers, the Decorators, Philadelphia 1960.

Burckhardt, J.: Die Kultur der Renaissance in Italien, 1860, 1958.

Burdach, $K$. (Hg.): Vom Mittelalter zur Reformation. Forschungen zur Geschichte der deutschen Bildung, 1893-1939.

ders.: Reformation, Renaissance, Humanismus. Zwei Abhandlungen über die Grundlage moderner Bildung und Sprachkunst, 1918.

Burger, H. O.: Renaissance Humanismus Reformation, 1969.

Cholevius, C. L.: Geschichte der deutschen Poesie nach ihren antiken Elementen, I, 1854.

Creizenach, W.: Geschichte des neueren Dramas, I, 1893-1911.

Eggers, H.: Deutsche Sprachgeschichte III. Das Frühneuhochdeutsche, 1969.

Ebrismann, G.: Geschichte der deutschen Literatur bis zum Ausgang des Mittelalters, 2,2,2, 1935.

Ellinger, G.: Geschichte der neulateinischen Literatur im sechzehnten Jahrhundert, I, 1929.

Entner, H.: Der Begriff "Humanismus « als Problem der deutschen Literaturgeschichtsschreibung, in: Klio 40, 1962, S. 260-270. (I).

ders.: Probleme der Forschung zum deutschen Frühhumanismus, in: WZUG, gesellschafts- und sprachwiss. Reihe 15, 1966, S. 587-590. (II).

ders.: Frühhumanismus und Schultradition in Leben und Werk des Wanderpoeten Samuel Karoch von Lichtenberg, 1968. (III).

Garin, E.: Der italienische Humanismus, Bern 1947.

ders. (Hg.): Prosatori latini del Quattrocento, Mailand-Neapel 1952.

Gebring, F. W.: Die Leistung deutscher Prosabearbeiter im Rahmen der Geistesgeschichte des 15. Jahrhunderts, in: Euphorion 34, 1933, S. 271-293.

Geiger, L.: Renaissance und Humanismus in Italien und Deutschland, 1882.

Gervinus, G. G.: Geschichte der poetischen National-Literatur der Deutschen, 2. Teil, 1836.

Gilmore, M. P.: The World of Humanism, New York 1942.

Goedeke, K.: Grundriß zur Geschichte der deutschen Dichtung, I, 1884, 2. Aufl.

Grossmann, K.: Die Frühzeit des Humanismus in Wien bis zu Celtis' Berufung 1497, in: Jb. f. Landesk. v. Niederösterreich, N. F. 22, 1929, S. 150-325.

Hagen, K.: Deutschlands literarische und religiöse Verhältnisse im Reformationszeitalter, 1841. 
Hankamer, P.: Die Sprache. Ihr Begriff und ihre Deutung im 16. und 17. Jahrhundert, 1927.

Hartfelder, $K$.: Deutsche Übersetzungen klassischer Schriftsteller aus dem Heidelberger Humanistenkreis, 1883/84.

Heger, H. (Hg.): Spätmittelalter, Humanismus, Reformation: Texte und Zeugnisse; 1. Teilbd.: Spätmittelalter und Frühhumanismus, 1975.

Heitz, P. und Ritter, F. J.: Versuch einer Zusammenstellung der deutschen Volksbücher des 15. und 16. Jahrhunderts, Straßburg 1924.

Herding, O.: Probleme des frühen Humanismus in Deutschland, in: AKG 38, 1956, S. 344-389.

Herrmann, M.: Albrecht von Eyb und die Frühzeit des deutschen Humanismus, 1893.

ders.: Die Reception des Humanismus in Nürnberg, 1898.

Hess, U.: Heinrich Steinhöwels »Griseldis «, 1975.

Joachimsen (auch -sobn), P.: Frühhumanismus in Schwaben, in: Württ. Vjhh. f. Landesgeschichte N. F. 5, 1896, S. 63-126; S. 257-288. (I).

ders.: Geschichtsauffassung und Geschichtsschreibung in Deutschland unter dem Einfluß des Humanismus, 1910. (II).

ders.: Humanismus und die Entwicklung des deutschen Geistes, in: DVjs 8, 1930, S. 419-480. (III).

ders.: Vom Mittelalter zur Reformation, in: Hist. Vjs. 20, 1920/21, S. 426-470; (als Buch 1959). (IV).

Karstien, C.: Beiträge zur Einführung des Humanismus in die deutsche Literatur, in: GRM 11, 1923, S. 217-225; S. 278-288.

König, E.: "Studia humanitatis* und verwandte Ausdrücke bei den deutschen Frühhumanisten, in: Beiträge z. Gesch. der Renaissance und Reformation. Festgabe J. Schlecht, 1917, S. 202-207.

Könneker, B.: Deutsche Literatur des Humanismus und der Reformation, in: Neues Handbuch der Literaturwissenschaft, X, 1972, S. $145-176$.

Kristeller, P. O.: The Classics and Renaissance Thought, Cambridge, Mass. 1955, 1961. (I).

ders.: Renaissanceforschung und Altertumswissenschaft, in: Forschungen und Fortschritte 33, 1959, S. 363-369. (II).

ders.: Renaissance Thought II. Papers on Humanism and the Arts, New York, Evanstown and London 1965. (III).

ders.: Der italienische Humanismus und seine Bedeutung, 1969.

Martin, A. v.: Soziologie der Renaissance, 1932, 1949.

Müller, G.: Deutsche Dichtung von der Renaissance bis zum Ausgang des Barock, 1927, 1957.

Muther, R.: Die deutsche Bücherillustration der Gotik und Frührenaissance (1460-1530), 1884.

Newald, R.: Probleme und Gestalten des deutschen Humanismus, hg. v. H.-G. Roloff, 1963. 
Oppermann, H. (Hg.): Humanismus, 1970.

Peuckert, W.-E.: Die große Wende. Das apokalyptische Saeculum und Luther, 1948, 1966.

Post, R. R.: The Modern Devotion, Leiden 1968.

Ristow, B.: Humanismus, in: RL 1, 1958, S. 693-727.

Ritter, G.: Die geschichtliche Bedeutung des deutschen Humanismus, in: HZ 127, 1923, S. 393-453.

ders.: Die Heidelberger Universität, I, 1936.

Rosenfeld, H.-F.: Humanistische Strömungen, in: Deutsche Wortgeschichte, hg. von Maurer-Stroh, I, S. 329-438.

Rüdiger, H.: Die Wiederentdeckung der antiken Literatur im Zeitalter der Renaissance, in: Geschichte der Textüberlieferung, hg. v. M. Meier, F. Hindermann und A. Schindler, 1961.

Rupprich, H.: Die deutsche Literatur vom späten Mittelalter bis zum Barock. 1. Teil: Das ausgehende Mittelalter, Humanismus und Renaissance (1370-1520), 1970. (I).

ders.: Die Frühzeit des Humanismus und der Renaissance in Deutschland, 1938, 1964 (Deutsche Literatur in Entwicklungsreihen). (II).

ders.: Humanismus und Renaissance in den Städten und an den Universitäten, 1935, 1964 (Deutsche Literatur in Entwicklungsreihen). (III).

Scherer, W.: Die Anfänge des deutschen Prosaromans, 1877.

Schmidt, Ch.: Histoire littéraire de l'Alsace à la fin du XVe et au commencement du XVIe siècle, 2 Bde, Paris 1879.

Schramm, A.: Der Bilderschmuck der Frühdrucke, 23 Bde, 1920 bis 1943.

Schwaderer, R.: Boccaccios deutsche Verwandlungen: Ubersetzungsliteratur und Publikum im deutschen Frühhumanismus, in: Arcadia 10, 1975, S. 113-128.

Schwarz, W.: Translation into German in the Fifteenth Century, in: MLR 39, 1944, S. 368-373. (I).

ders.: Theory of Translation in 16th Century Germany, in: MLR 40, 1945, S. 289-299.

Seidlmayer, M.: Wege und Wandlungen des Humanismus, 1965.

Spitz, L. W.: The Religious Renaissance of the German Humanists, Cambridge, Mass., 1963.

Stammler, W.: Von der Mystik zum Barock, 1927, 1950. (I).

ders.: Zur Sprachgeschichte des 15. und 16. Jahrhunderts, in: Vom Werden des deutschen Geistes, Festgabe G. Ehrismann, hg. v. W. Stammler, 1925. (II).

ders. und $K$. Langosch: Die deutsche Literatur des Mittelalters. Verfasserlexikon, 5 Bände, 1933-1955.

Störig, H. J.: Das Problem des Ubersetzens, 1969.

Strauch, P.: Pfalzgräfin Mechthild in ihren literarischen Beziehungen, 1883.

Toepke, G. (Hg.): Matrikelverzeichnis der Universität Heidelberg, 1. Teil: Von 1386-1662, 1884. 
Voigt, G.: Die Wiederbelebung des classischen Alterthums, 2 Bde, 1859, 1893. (I).

ders.: Enea Silvio de'Piccolomini als Papst Pius II und sein Zeitalter, 3 Bde, 1856-1863. (II).

Weil, E.: Der Ulmer Holzschnitt im 15. Jahrhundert, 1923.

Wenzlau, F.: Zwei- und Dreigliedrigkeit der deutschen Prosa des XIV. und XV. Jahrhunderts, 1906.

Worstbrock, F. J.: Zur Einbürgerung der Ubersetzung antiker Autoren im deutschen Humanismus, in: ZfdA 99, 1970, S. 45-81.

Wuthenow, $R$. R.: Das fremde Kunstwerk. Aspekte der literarischen Übersetzung, 1969. 